measure of their potential, at least in WGTA type situations which are adequate to produce ODLS formation in bluejays and mynas. The crows seemed well motivated, consistently responding on all trials during a session and consuming all available reinforcements. They did solve individual problems, and the results of the hypothesis analysis shows that they were not responding randomly, but systematically.

These results should be extended through further research. If they represent an accurate assessment of the ability of crows to form a visual learning set, then large differences in ODLS performance exist within a single family. This would suggest that comparative studies of learning might profitably investigate learning in closely related species. Such results would also be counter to Gossette's (1974) taxonomic distance hypothesis, which holds that closely related species, such as members of the same family, should show more similar learning performances than distantly related species. At the present, the ODLS behavior of one corvid, the bluejay, appears to be more similar to that of a sturnid, the myna, than to another corvid, the crow. tasks. Psychological Bulletin, 1967, 67, 87-92.

Gossette, R. L. Note on methodology in comparative behavioral analysis: systematic variation vs. control by equation. Perceptual and Motor Skills, 1974, 39, 155-162.

Harlow, H. F. Learning set and error factor theory. In S. Koch (Ed.), Psychology a study of a science. New York: McGraw Hill, 1959, Vol. 2, Pp. 492-537.

Hunter, M. W., \& Kamil, A. C. Object-discrimination learning set and hypothesis behavior in the northern bluejay (Cyanocitta cristata). Psychonomic Science, 1971, 22, 271-273.

Kamil, A. C., \& Hunter, M. W. Performance on object-discrimination learning set by the Greater Hill Myna (Gracula religiosa). Journal of Comparative and Physiological Psy chology, 1970, 73, 68-73.

Levine, M. A model of hypothesis behavior in discrimination learning-sets. Psy chological Review, 1959, 66, 353-366.

Levine, M. Hypothesis behavior. In A. M. Schrier, H. F. Harlow, and F. Stollnitz (Eds.), In behavior of nonhuman primates: Modern research trend8. Vol. 1, New York: Academic Press, 1965, Pp. 97-127.

Powell, $R$. W. Comparison of differential reinforcement of low rates (DRL) performance in pigeons (Columba livia) and crows (Corvus brachyrhynchos). Journal of Comparative and Physiological Psychology, 1974, 86, 736-746.

\title{
REFERENCES
}

Fellows, B. J. Chance stimulus sequences for discrimination

(Received for publication January 17, 1975.)

Bulletin of the Psychonomic Society

1975, Vol. 5 (5), 375-377

\section{Human evoked brain responses following loud pure tones}

\author{
JAMES K. WALSH and DONALD I. TEPAS \\ Saint Louis University, Saint Louis, Missouri 63103
}

\begin{abstract}
The electroencephalogram (EEG) was recorded at the vertex of three subjects following a 5-min exposure to a $720-\mathrm{Hz}$ pure tone at $110 \mathrm{~dB}$ or $45 \mathrm{~dB}$ SPL. Eight consecutive evoked brain responses (EBRs) to tone bursts were computed from the EEG for a 2-min period following each exposure. The EBRs computed following the 110-dB exposure were initially smaller than those computed following the 45-dB exposure, and this difference became minimal as time after exposure increased. Thus, EBR amplitude data suggest that a temporary threshold shift (TTS) was produced by the 110-dB exposure and that the EBR reflects this change in the listener's sensitivity.
\end{abstract}

A reversable elevation in hearing threshold following exposure to an auditory stimulus is frequently referred to as a temporary threshold shift (TTS). Generally, the difference between a listener's threshold for a standard test signal before and after exposure defines the degree of TTS. Although considerable TTS research is reported in the literature (Miller, 1974; Ward, 1963), little comparable electrophysiological data is evident. Human electrophysiological data are limited to a single brief report of EBR threshold estimations from a single listener following over $24 \mathrm{~h}$ of octave band noise exposure. These data suggest that EBR changes similar to those measured behaviorally are present (Mills,

Requests for reprints should be sent to James $K$. Walsh, Department of Psychology, Saint Louis University, St. Louis, Missouri 63103.
Gengel, Watson, \& Miller, 1970). Since extensive research from numerous laboratories has established the relation of human EBR measures to basic acoustic parameters (Davis \& Zerlin, 1966; McCandless \& Best, 1966; Rapin, Schimmel, Tourk, Krasnegor, \& Pollack, 1966; Tepas, Boxerman, \& Anch, 1972), the observation of Mills et al. (1970) appears reasonable. The present experiment is a further confirmation that human EBR measures are sensitive to noise exposure conditions with known TTS-producing characteristics.

Specifically, we proposed that the amplitudes of EBRs computed immediately following noise exposure would be smaller when exposure conditions approached TTS levels. Using the psychophysical study of Mills and Lilly (1971) as a guide, EBRs computed following a probable TTS-producing exposure were compared with 
those computed immediately following a control exposure condition. The exposure conditions used were drastically shorter than that used by Mills et al. (1970) to evaluate the feasibility of detecting the effects of fairly modest durations of exposure using EBR measures. Differences in the amplitude of the EBR produced by these two exposure conditions provided additional evidence that this electrophysiological measure is sensitive to traditional psychoacoustic variables.

\section{METHOD}

\section{Subjects}

Three male graduate students in psychology served as listeners. The mean age of these volunteers was 25 years. Each of the listeners had previous experience as a subject in EBR research. All of the listeners had normal hearing as tested by an Ambco Model A-17 audiometer prior to the start of experimentation. The listeners were audiometrically tested aperiodically between experimental sessions for possible indications of hearing loss during the time course of the experiment. These precautionary procedures yielded negative results.

\section{Apparatus \\ A Jackson audio-oscillator, Model 655, produced pure exposure tone (ET) stimuli of $720 \mathrm{~Hz}$. These ET stimuli were led through Grason-Stadler 1200 modules which controlled and delivered the output through a single TDH-39 earphone. Monaural test signals (TS) were 100 -msec tone bursts of $1000 \mathrm{~Hz}$ having 10 -msec rise-fall times. A TS intensity of $80 \mathrm{~dB}$ SPL (re: $20 \mu \mathrm{N} / \mathrm{m}^{2}$ ) was chosen to insure an adequate signal-to-noise ratio in the EEG. TS were presented at a $1-\mathrm{sec}$ rate. A Hewlett-Packard Function Generator, Model HrssuUA, and Series 1200 modules determined the TS characteristics. \\ A monopolar recording was made by referring a Grass E5S electrode at $C_{z}$ (Jasper, 1958) to an $11-\mathrm{mm}$ Beckman biopotential electrode on the mastoid (contralateral to stimulus presentation). A 16-mm Beckman electrode on the forehead served as the ground. The EEG was amplified and recorded using a Grass Model 7 polygraph equipped with P5A preamplifiers. The polygraph driver amplifiers fed the amplified potentials to the AX08 analog input of a LAB-8 computer system. The one-half amplitude bandpass of the amplifiers at output to the computer was .15 to $500 \mathrm{~Hz}$, as specified by the manufacturer. EEG samples were collected from 125 data points distributed evenly over a $300-\mathrm{msec}$ period of analysis which began $20 \mathrm{msec}$ before each TS presentation. These samples were computed by the LAB-8 PDP-8I computer using the advanced averager program (DEC-LB-U18C-PB). At the beginning of each session, $10 \mu \mathrm{V}$ calibration signals produced by a Grass SD5 stimulator were similarly averaged to provide a calibration for measurements.}

\section{Procedure}

Each listener was tested on 15 occasions at approximately the same time of day. For each of these sessions, the listener was seated in an adjustable dental chair in a dark, electrically shielded chamber, and instructed not to adjust the earphones after the beginning of a trial. Two listeners were tested using the left ear for all sessions, while the third listener was tested using the right ear seven times and the left ear for eight sessions. Two levels of ET, $45 \mathrm{~dB}$ and $110 \mathrm{~dB}$ SPL, were used. ET duration for both levels was $5 \mathrm{~min}$. Exposures to $110 \mathrm{~dB}$ for $10 \mathrm{~min}$ at this frequency have been shown to produce a TTS (Mills and Lilly, 1971). Psychophysical research indicates that to produce a TTS in a normally hearing person, a stimulus must be at least $60 \mathrm{~dB}$ and perhaps as much as $80 \mathrm{~dB}$ even when exposure exceeds $8 \mathrm{~h}$ (Mills et al., 1970). Thus, the 45-dB ET was viewed as a control condition. Each session included exposure to both ET levels. The order of the two exposures within a session was randomized across sessions with each intensity first at least seven times. A 20-min rest period separated the two trials of each session.

Immediately upon the conclusion of each 5-min exposure, eight consecutive EEG samples to the TS were computed. Each of these samples was the sum of the EEG activity following 15 consecutive TS presentations and therefore represents a specific 15 -sec period following the exposure. Thus, the total averaging time for the eight EEG samples was 2 min. Using the LAB-8 contingency register, the experimenter controlled the storage location of this EEG information. EEG input was switched into successive contingency registers immediately after each 15 TS presentations.

The listener's EEG was monitored on the polygraph intermittantly during the 5-min ETs and continuously from a point $30 \mathrm{sec}$ prior to the offset of the ET until averaging was completed. A small red light was flashed in the recording chamber upon the beginning of continuous EEG monitoring. This flash indicated to the listener that averaging would begin in $30 \mathrm{sec}$ and that he should minimize body movements and other behaviors which might increase the noise in the EEG recordings.

During the rest period as well as after the second trial, the eight EEG samples were punched on paper tape to allow off-line pooling of EEG samples across sessions using the APE program (Tepas, Kress, \& Klingaman, in press). This cross-session summation of EEG samples yielded an EBR waveform for each of the eight post-ET time periods following both ET levels for each listener. Each EBR waveform represents the EEG response to 225 TS presentations.

\section{RESULTS}

EBR waveforms were plotted on an X-Y analog recorder after cross-session summation and the corresponding digital values representing the analog EBR waveforms were printed out on a ASR-33 teletype. Peak amplitude and latency measures were made from this digital data and calibrated using the appropriate digital values for the $10 \mu \mathrm{V}$ summed calibrations. Figure 1 shows the X-Y plots of all EBR waveforms obtained in this study from one listener. Similar waveform shapes for EBRs after both levels of exposure are evident. Prominent peaks and troughts have been arbitrarily labeled alphabetically following the nomenclature and rationale of Tepas (1974). An intermittently occurring negative deflection with a latency between $20-45 \mathrm{msec}$ was labeled $A$. A following postive voltage change at 40-60 msec was termed $B$. Neither Trough $A$ nor Peak $B$ occurred consistently in all the waveforms. A pronounced negative deflection around $70-115 \mathrm{msec}$ was called $C . D$ was the largest positive deflection with a latency of $155-220 \mathrm{msec}$. The next trough was termed $E$ and had a latency of about $250 \mathrm{msec}$. Peak-to-peak amplitude measurements, $A-B, B-C, C-D$, and $D-E$, and latency measurements for all five deflections were made when possible. Amplitude measures were most easily and consistently made for the $C$ - $D$ measure.

The data were pooled across listeners. Figure 2 shows mean $C-D$ amplitude values at both ET levels plotted as a 
function of the logarithm of the time after ET cessation. A clear difference in amplitude is indicated between EBRs computed after the 110-dB ET and the temporally corresponding EBRs following the 45-dB ET for at least $1 \mathrm{~min}$. As time after ET increases, the EBRs computed after the 110-dB ET and after the 45-dB ET generally become more similar in amplitude. This general trend was evident in the data of two of the individual listeners.

\section{DISCUSSION}

Inspection of Figure 2 suggests that a simple interpretation of the differences between the 45-dB ET and the 110-dB ET EBR data solely on a TTS effect may not be warranted. An alternative explanation could be based upon a possible differential habituation effect. If habituation is defined as decreasing response amplitude duing the repetitious presentation of a stimulus, this effect is suggested by the 45-dB ET data. It is not completely clear if the initial EBR amplitude reduction after the $110-\mathrm{dB}$ ET is due to a TTS in the traditional sense or whether the exposure causes some alteration in the process of habituation. It is reasonable to suggest that both TTS and habituation are important factors. We suggest that recovery from TTS and habituation to the TS are simultaneously occurring phenomena following ET. Since habituation and recovery from TTS most likely have opposite effects upon EBR amplitude, it is reasonable to suspect some cancellation by the two processes. This interpretation may explain the failure of the 110-dB ET data in Figure 2 to strongly suggest either an habituation or a recovery effect.

The results of this study clearly indicate that the EBR reflects changes produced by exposure to loud sounds. A more definitive statement is premature and should be withheld awaiting further research. The small sample size and the possible compunding effect of habituation contribute to the limited nature of our conclusions and an accurate estimation of EBR functional relationships is probably difficult and inappropriate. This does not necessarily mean that the EBR is an insensitive indicant of TTS. It may merely be an indication that EEG signal averaging requires relatively large samples to extract EBR data free from contaminative EEG variability which is not correlated with stimulation parameters (Tepas, 1974). Unfortunately, the collection of large samples of data in an EBR-TTS study is difficult and time consuming. Caution must be taken when exposing humans to intense stimuli for extended periods of time and the number of exposures should be minimized. In addition,

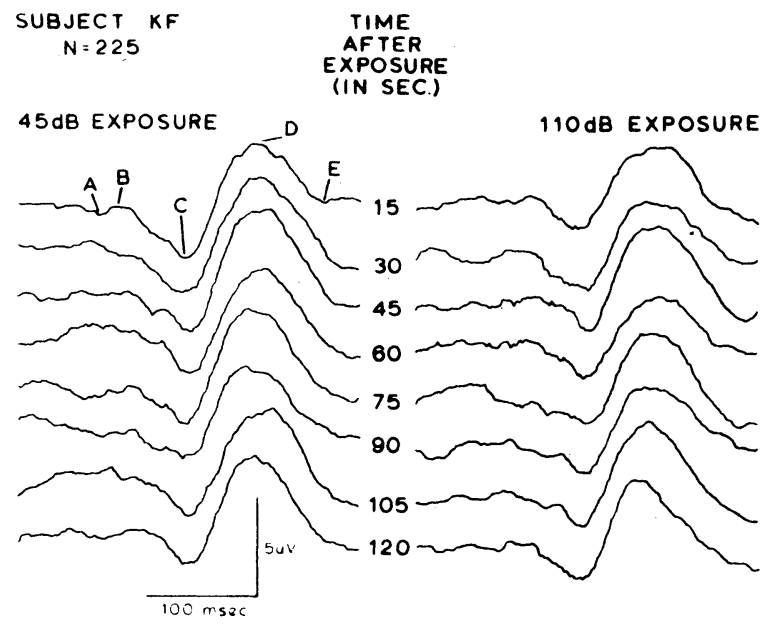

Figure 1. All evoked brain response waveforms recorded from listener KF. Peaks and troughs are labeled alphabetically in order of their occurrence. This listener was tested using the left ear for all sessions.

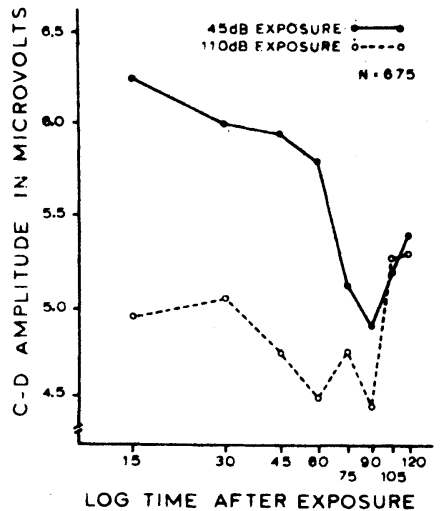

IN SECONDS

Figure 2. Mean C-D amplitudes for three listeners plotted as a function of the logarithm of time after exposure.

TS presentation and EBR averaging must be limited to prevent significant confounding of TTS by the TS itself.

Finally, the present data lend support to a few methodological techniques for auditory EBR research in general. Sound exposures immediately before computation of EBRs has been shown to greatly effect the amplitude of EBRs in the present study. Whether or not this difference is due to recovery from a loud sound or some other factor(s), immediate exposure history should be considered in the design and execution of most auditory EBR studies. The systematic use of adequate intertrial intervals is suggested. In addition, stimulus randomization procedures must consider these exposure effects. Simple randomization may not be adequate if not accompanied by appropriate consideration of sample size and other factors.

\section{REFERENCES}

Davis, H., \& Zerlin, S. Acoustic relations of the human vertex potential. Journal of the Acoustical Society of America, 1966 39, 109-116.

Jasper, $H$. The ten-twenty electrode system of the international federation. Electroencephalography and Neurophysiology, 1958, 10, 371-375.

McCandless, G., \& Best, L. Summed evoked responses using pure tone stimuli. Journal of Speech and Hearing Research, 1966 9, 266-272.

Miller, J. The effect of noise on man. Journal of the Acoustical Society of America, 1974, 56, 729-764.

Mills, J., Gengel, R., Watson, C., \& Miller, J. Temporary changes of the auditory system due to exposure to noise for one or two days. Journal of the Acoustical Society of America, 1970, 48, 524-530.

Mills, J., \& Lilly, D. Temporary threshold shifts produced by pure tones and noise in the absence of an acoustic reflex. Journal of the Acoustical Society of America, 1971, 60, 1556-1558.

Rapin, I., Schimmel, H., Tourk, L., Krasnegor, N., \& Pollack, C. Evoked responses to clicks and tones of varying intensity in waking adults. Electroencephalography and Clinical Neuro physiology, $1966,21,335-344$.

Tepas, D. I. Computer analysis of the electroencephalogram: Evoking, promoting, and provoking. Behavior Research Methods \& Instrumentation, 1974, 6, 95-1 10.

Tepas, D. I., Boxerman, L. A., \& Anch, A. M. Auditory evoked brain responses: Intensity function from bipolar human scalp recordings. Perception \& Psychophysics 1972, 11, 217-221.

Tepas, D. I., Kress, G., \& Klingaman, R. L. APE: Average Potential Evaluation Software for the LAB-8 System. Behavior Research Methods \& Instrumentation, 1975, 7, in press.

Ward, W. 'D. Auditory fatigue and masking. In J. Jerger (Ed.), Modern developments in audiology. New York, New York Academic Press, 1963.

(Received for publication Januarv 24,1975 .) 